\title{
Case-study of Localization via WSN Using Distributed Compressed Sensing
}

\author{
Veronika Olešnaníková, Michal Kochláň, IEEE Student Member, Róbert Žalman \\ University of Žilina \\ Faculty of Management Science and Informatics, \\ Univerzitná 8215/1 Žilina 01026 \\ Email: \{Michal.Kochlan, Robert.Zalman, Veronika.Olesnanikova\}@fri.uniza.sk
}

\begin{abstract}
Distributed compressed sensing task can be parallelized into several nodes that is highly suitable for using in Wireless Sensor Networks. Localization is one of the critical tasks solved in wireless systems. This paper investigates the possibilities of localization using compressed sensing implemented on wireless nodes and aggregation node. The presented case study simulates the application scenario of a target deployed in the field. This target is being localized by the wireless sensor network based on the emitted acoustic signal. Several types of the emitted signals have been used during the simulation runs. The emphasis was put on the properties of the reconstruction process such as compression ratio and minimization of the reconstruction error.
\end{abstract}

\section{INTRODUCTION}

$\mathbf{T}$ HE PROBLEM of target localization, in general, can be defined as finding an object in the space. Localization process in wireless sensor networks aims to localize a target based on the sensor data from the spatially distributed wireless nodes. For a single target and/or source localization in wireless sensor networks, there are various methods. For outdoor localization, this can include localization in traffic monitoring (vehicles, aircraft, bicycles, etc.). In indoor environment persons as well as animals and object can be tracked. Scientific literature refers to two core ways for estimation of the target location:

- Angle of Arrival (AOA) [1];

- Time Difference of Arrival (TDOA) [2-5].

In this paper, we are focused on the algorithms for singlesource localization. The literature recognizes two basic groups of these algorithms:

- Energy Decay Model-based Localization Algorithms (EDMLA);

- Model Independent Localization Algorithms (MILA).

\section{A. Decay Model-Based Method}

The following formula shows the decay model which is in detail described in [6-8]. The received signal strength at i-th wireless node in time instant $t$ can be expressed as follows:

$$
y_{i}(t)=g_{i} \frac{E(t)}{d_{i k}^{2}(t)}+n_{i}(t),
$$

where $g_{i}$ means gain factor of i-th sensor. $E(t)$ is the energy of the received signal in 1 meter distance from the wireless node, and $d_{i k}$ is the Euclidean distance between $i$-th sensor and the target. Moreover, $n_{i}$ represents measurement noise with zero mean value and Gaussian probability distribution with variance $\sigma_{i}^{2}$, i.e. $N\left(0, \sigma_{i}^{2}\right)$.

\section{B. Model-Independent Method}

Authors in [9] describe a kernel averaging approach which does not need information about energy decay model. On the other hand in [10], the authors propose novel modelindependent localization method and employed a distributed sorting algorithm. The research relies on the fact that the nodes closer to the target can measure higher RSSI (received signal strength indicator). Assuming that the wireless nodes know their rank, the distance estimates can be calculated from the respective probability density functions. RSSI indicator at i-th sensor in time instant $t$ is as follows:

$$
y_{i}(t)=g_{i} \sum_{k=1}^{K} \frac{E_{k}(t)}{d_{i k}^{\alpha}(t)}+\epsilon_{i}(t),
$$

where $d_{i k}(t)$ is the distance between the $i$-th sensor and $k$-th target. $K$ is the number of targets. $g_{i}$ is gain of $i$-th sensor. $\epsilon_{i}(t)$ is random variable with mean value equal to $\mu_{i}$ and variance given as $\sigma_{i}^{2} . E_{k}(t)$ is the energy of the received signal in 1 meter distance from $k$-th target. $\alpha$ represents attenuation exponent.

The target localization task can be performed by the distributed nodes of a wireless network. An interesting approach arises from combination of compressed sensing and wireless sensor networks using distributed compressed sensing.

\section{Distributed Compressed SEnsing}

A wireless sensor network (WSN) is formed by numerous spatially distributed devices (nodes) that process sensor data. Each node has a power source e.g. in form of battery thus having a limited lifetime. Since the energy consumption is a critical point, low power and efficient signal processing units are used in wireless nodes. Thus, wireless sensor nodes have limited computing and communication capabilities. Although, these nodes have low individual computing power, they can cooperate so that the computational power of the whole network allows performing advanced signal processing tasks [11]. Having the ability of advanced processing tasks leads to higher degree of robustness and greater versatility in low-lost scenario. This represents one of the most attractive reasons 
why WSNs are used for wide range of remote sensing and environmental monitoring applications [12].

From the point of signal processing theory, a major challenge in WSNs is effective design of set of sensor-local signal processing operations and strategies suitable for intersensor communication and networking in order to address the desired trade-off among energy consumption, simple design, and overall system performance [13]. This trade-off shows, for example, in sensor lifetime maximization and effective battery utilization when reducing communication bandwidth. This can be achieved by each sensor by locally compressing the observed data and thus low rate inter-sensor communication is required. Such techniques can be represented by distributed compressed sensing (DCS).

Typical DCS scenario comprises numerous sensors measuring individually sparse signals, which are correlated among each other [14]. It should be noted that the signals are sparse in a certain basis. Each sensor individually encodes its signal by transforming it into another, incoherent basis (for example a random one). Then the sensor broadcasts only a few of the resulting coefficients to the aggregation node [14]. One of the advantages of DCS is that it does not require collaboration among the sensors when obtaining and processing the signal. Moreover, random projections in DCS are universal [15]. This means that any sparse basis can be used, which allows the same encoding strategy to be applied in different scenarios. This contributes to the robustness of the solutions based on DCS, i.e. the measurement stream from each sensor has equal priority. This is different from Fourier or wavelet transforms It should be also mentioned that random measurements allow a progressively better recovery of the data, that means single measurement or more can be lost without the effect on the entire recovery process.

The problem of DCS illustrates the described example that follows. Let's have a network of $n$ nodes, where each node has a piece of information given by $x_{j}$ where $j=1, \ldots, n$. Let's assume that each piece of information $x_{j}$ is a scalar quantity. Together, the scalar quantities form a data vector $\boldsymbol{x}=$ $\left[x_{1}, \ldots, x_{n}\right]^{T}$, which is called networked data. This underlines the fact that the data is distributed across the network and that the data may be shared over the network [17].

In wireless sensor networks, $n$ can be a large number, thus having the networked data large as well. Therefore, the process of data acquisition at a single point is daunting. However, let's imagine that it is possible to create highly compressed version of vector $\boldsymbol{x}$ in a decentralized fashion. Scientific literature states several decentralized compressed sensing strategies. One strategy relies on the correlations among the a priori known data at different nodes [21]. In such case, a technique called distributed source coding known as Slepian-Wolf coding can be utilized as a compression scheme that allows none or little collaboration among the nodes. However, in lots of application scenarios the prior knowledge of the data correlations is not known. This situation supports research in collaborative signal processing within the sensor networks as well as data compression techniques [20]. It can be quite challenging to propose and implement an effective algorithm of distributed and collaborative processing for wireless sensor network [22]. Such algorithms rely to a great extend on specific prior knowledge and the relation of the expected signal correlations. The success of the implementation of such algorithms lies in sophisticated communication pattern and good processing capabilities of a sensor node.

The mentioned projections in standard multi-hop wireless networks utilizing compressed sensing can be expressed as vector $\boldsymbol{y}$, which components $y_{i}$ can be calculated as follows:

$$
y_{i}=\sum_{j=1}^{n} A_{i, j} x_{j} .
$$

The components $y_{i}$ are able to be computed in a decentralized fashion and efficiently because each value of the compressed data is represented as a simple linear combination of the values obtained at each node [23].

Basically, there are two simple steps in the computation and transmission of each compressed data sample $y_{i}$, where $i=1, \ldots, k$ [23]:

1) Let's consider $n$ sensor nodes in the wireless sensor network. Each of the sensors as its index $j=1, \ldots, n$. Each node $n_{j}$ computes locally properties $A_{i, j}$ and $x_{j}$ so that the measured data are being multiplied with the corresponding element of the measurement matrix. The measurement matrix can be distributively created as local (at node) realization of $A_{i, j}$ using a pseudo-random number generator initialized by the node identifier, e.g. integers $j=1, \ldots, n$. Having these node identifiers, particular node can simply calculate the vectors $\left\{A_{i, j}\right\}_{i=1}^{k}$, where sensors are indexed as $j=1, \ldots, n$.

2) The local sensor node variables $A_{i, j} x_{j}$ are being continuously combined and transmitted over the sensor network using so called randomized gossip. The randomized gossip represent a decentralized algorithm, which computes linear functions such as:

$$
y_{i}=\sum_{j=1}^{n} A_{i, j} x_{j} .
$$

To summarize compressed sensing, one could say that it is a technique for signal processing and signal representation, where the signal can be sensed only by such number of samples, which corresponds to the signal sparsity in some base. The overall nature of compressed sensing matches the nature of event-driven control presented in the previous sections. Event-driven signal representation and reconstruction mechanism also leads to signal sampling and its reconstruction by as many (little) samples as are truly needed.

\section{CASE Study AND Numerical Results}

The idea of the localization system is to identify the position of the target by multiple WSN nodes deployed in the area. To decrease the consumption of the nodes the compressed sensing algorithms are supposed to be used. In order to use DCS the transmitted signal by the target has to follow special 


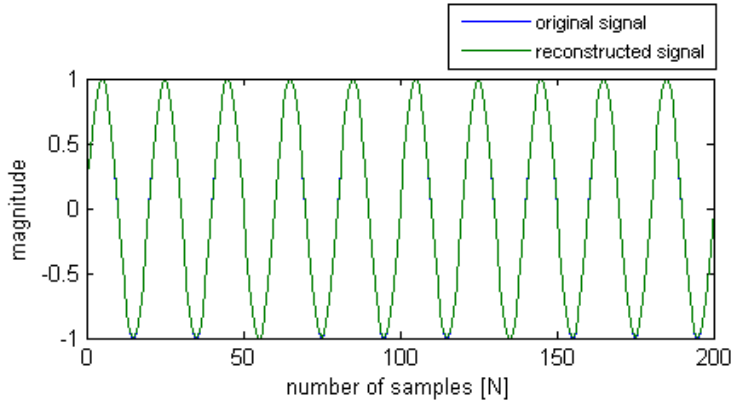

Figure 1. Continuous signal before and after reconstruction

requirements, e.g. certain sparsity in the frequency domain. This paper investigates signals in acoustic domain, i.e. in rage $20 \mathrm{~Hz}$ up to $20 \mathrm{kHz}$.

For the simulation purposes, the Matlab version 2013b was used. Reconstruction of original signal was performed by using the L1-magic library. The main goal of this article was to inspect the properties of different signals which could be used in the localization tasks. These signals should have suitable parameters for the reconstruction in order to use compressed sensing. All used signals are sparse in the frequency domain.

In the first simulation case, the signal was continuously transmitted. The signal has the sinusoid shape with the frequency of $100 \mathrm{~Hz}$. The reconstruction using the distributed compressed sensing performs well with compression ratio (cr see equation (5)) up to 200. The compression ratio is expressed by the following formula:

$$
c r=\frac{s_{a}}{\chi},
$$

where $\mathrm{cr}$ is the compression ratio, $s_{a}$ represents the number of all samples in the original signal and $\chi$ is the number of randomly selected compressed sensing coefficients. The following Fig. 1 shows the reconstruction of harmonic signal $100 \mathrm{~Hz}$. Despite the fact that in the reconstruction of such a signal the compression ratio can be relatively high, this class of signals are not suitable for localization purposes. The reason is that we are not able to identify the same particular part of the received signal, e.g. the start of the same period.

The second simulation scenario is based on the transmitting the signal in bursts. By modification of the above-mentioned continuous signal, we are able to detect the start of the burst, thus, all nodes are able to determine the time of arrival of the received signal.

The Fig. 3 depicts the reconstruction of the burst signal with the same compression ratio as in the Fig. 1. The burst signal is compounded of the carrier signal and a secondary frequency. Carrier signal has a frequency of the $100 \mathrm{~Hz}$ and secondary modulated signal has a frequency of $500 \mathrm{~Hz}$. The burst contains two periods of the carrier and ten periods of the secondary signal. Having this compression ratio the reconstruction results are insufficient for localization purposes.

Proper change of the parameters of the compression ratio leads to reconstruction improvement. This is demonstrated on

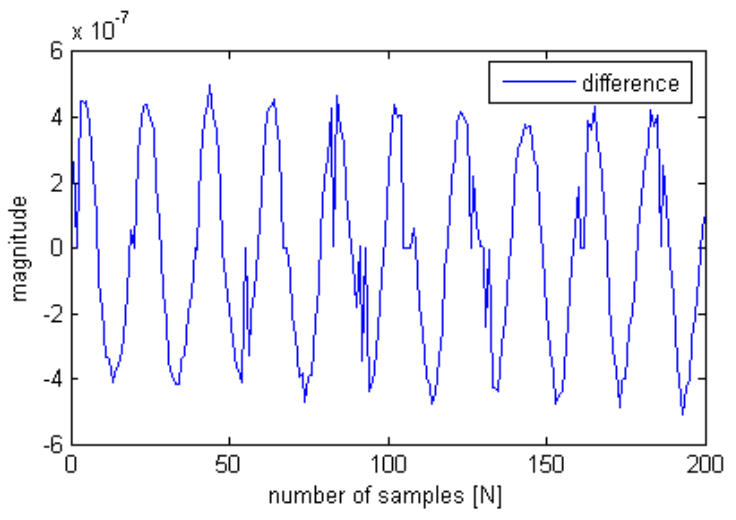

Figure 2. Difference of original and reconstructed continuous signal, cr=100

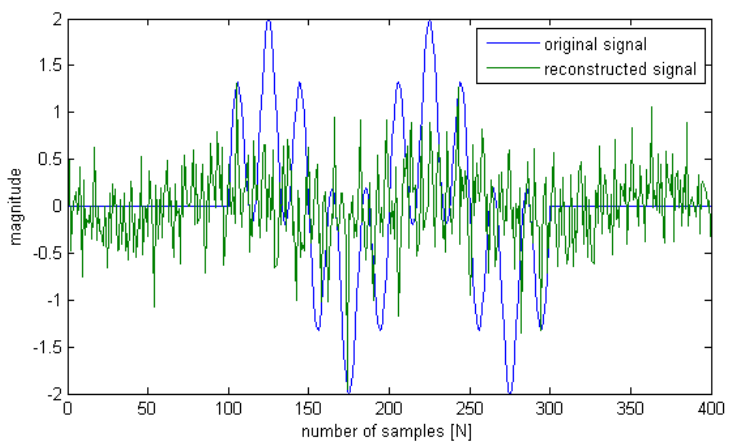

Figure 3. Burst signal (BURST01) before and after reconstruction

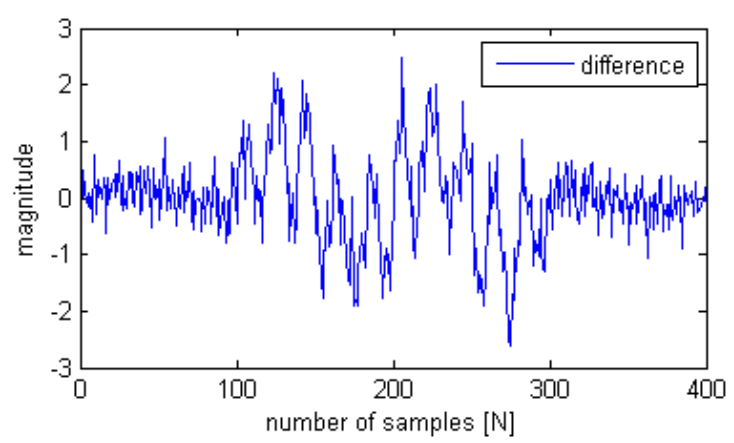

Figure 4. Difference of original and reconstructed burst signal, cr=100

the Fig. 5, along with difference between the original and the reconstructed signal on the Fig. 6. Compression ratio was decreased from 100 to 20 .

Sumarization of the parameters used in the simulation is shown in Table I. It is obvious that continuous signals are very suitable for compressed sensing and can be reconstructed using high compression ratio, however, it is difficult to use them for localization purposes. The reconstruction error was calculated as a mean value based on differences between the original and reconstructed signals (see Fig. 2, 4, 6). 


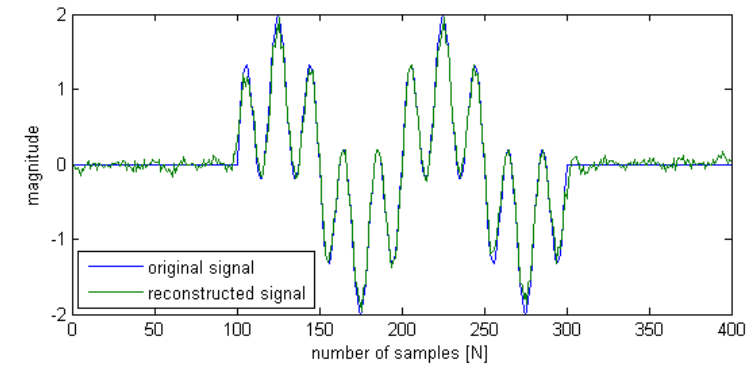

Figure 5. Burst (BURST02) signal before and after reconstruction

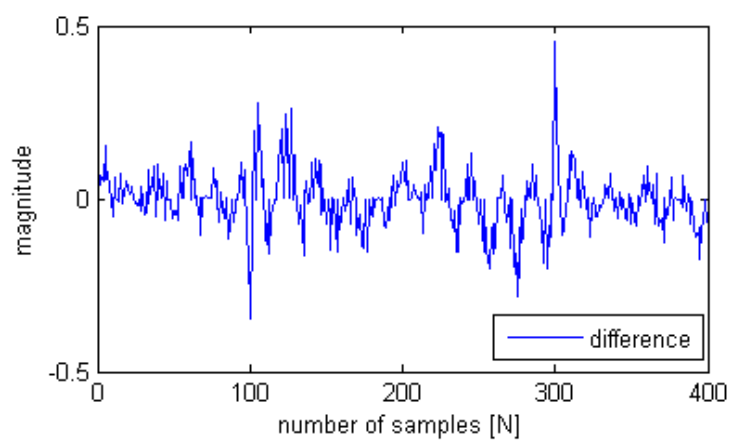

Figure 6. Difference of the original and reconstructed burst signal, $\mathrm{cr}=20$

Table I

PARAMETERS AND THE RESULTS OF THE SIMULATIONS

\begin{tabular}{|l|c|c|c|c|}
\hline $\begin{array}{c}\text { Type } \\
\text { of the } \\
\text { signal }\end{array}$ & Frequency & $\begin{array}{c}\text { Number } \\
\text { of } \\
\text { periods }\end{array}$ & cr & Error \\
\hline CONTINUOUS & $100 \mathrm{~Hz}$ & 10 & 100 & $9.52 \cdot 10^{-8}$ \\
\hline BURST01 & $100 \mathrm{~Hz}$ & 10 & 100 & 0.5638 \\
\hline BURST02 & $100 \mathrm{~Hz}$ & 10 & 20 & 0.0536 \\
\hline
\end{tabular}

\section{CONCLUSION}

This contribution investigates suitable signals for target localization using WSN and DCS. The results show, that continuous periodic signals can be reconstructed easily with high compression ratio using DCS. However, these signals are not target-identifiable. Changing the form of the transmitted signal broadcast from the target enables the sensor network to detect it. The changed signal has the form of burst, which enables to detect the start of the beacon signal. Based on the time of the arrival and the ability to detect the start of the beacon signal, the network is able to localize the target.

\section{REFERENCES}

[1] L. M. Kaplan, Q. Le, and P. Molnar, "Maximum likelihood methods for bearings-only target localization", in Proceedings of IEEE International
Conference on Acoustics, Speech, and Signal Processing, vol. 5, pp. 3001 - 3004, Salt Lake City, Utah, USA, May 2001.

[2] Y. Weng, W. Xiao, and L. Xie, "Total least squares method for robust source localization in sensor networks using TDOA measurements", International Journal of Distributed Sensor Networks, vol. 2011, Article ID 172902, 8 pages, 2011.

[3] X. Qu and L. Xie, "Source localization by TDOA with random sensor position errors-part I: static sensors", in Proceedings of the 15th International Conference on Information Fusion, pp. 48-53, Singapore, July 2012.

[4] X. Qu and L. Xie, "Source localization by TDOA with random sensor position errors-part II: mobile sensors", in Proceedings of the 15th International Conference on Information Fusion, pp. 54-59, Singapore, July 2012

[5] K. C. Ho, "Bias reduction for an explicit solution of source localization using TDOA", IEEE Transactions on Signal Processing, vol. 60, no. 5, pp. 2101-2114, 2012

[6] D. Blatt and A. O. Hero, "Energy-based sensor network source localization via projection onto convex sets", IEEE Transactions on Signal Processing, vol. 54, no. 9, pp. 3614-3619, 2006.

[7] K. Deng and Z. Liu, "Weighted least-squares solutions of energy-based collaborative source localization using acoustic array", International Journal of Computer Science and Network Security, vol. 7, no. 1, pp. 159-165, 2007.

[8] Q. Shi and C. He, "A new incremental optimization algorithm for MLbased source localization in sensor networks", IEEE Signal Processing Letters, vol. 15, pp. 45-48, 2008.

[9] M. G. Rabbat, R. D. Nowak, and J. Bucklew, "Robust decentralized source localization via averaging", in Proceedings of IEEE International Conference on Acoustics, Speech, and Signal Processing (ICASSP '05), vol. 5, pp. V1057-V1060, Philadelphia, Pa, USA, March 2005.

[10] D. Ampeliotis and K. Berberidis, "Energy-based modelindependent source localization in wireless sensor networks," in Proceedings of the 16th European Signal Processing Conference, Lausanne, Switzerland, August 2008.

[11] I.F. Akyildiz and W. Su and Y. Sankarasubramaniam and E. Cayirc, "Wireless sensor networks: a survey", in Computer Networks, 2002, ISSN: 1389-1286

[12] A. Mainwaring and D. Culler and J. Polastre and R. Szewczyk and J. Anderson, "Wireless sensor networks for habitat monitoring", in Proceedings of the 1st ACM international workshop on Wireless sensor networks and applications, 2002

[13] M. A. Razzaque and Ch. Bleakley and S. Dobson, "Compression in wireless sensor networks: A survey and comparative evaluation", in ACM Transactions on Sensor Networks (TOSN), 2013

[14] C. Caione and D. Brunelli and L. Benini, "Distributed compressive sampling for lifetime optimization in dense wireless sensor networks", in Industrial Informatics, IEEE Transactions on, 2012

[15] K. Hayashi and M. Nagahara and T. Tanaka, "A user's guide to compressed sensing for communications systems", in IEICE transactions on communications, 2013

[16] S. Foucart and H. Rauhut, "An Invitation to Compressive Sensing", 2013

[17] Y. C. Eldar and G. Kutyniok, "Compressed sensing: theory and applications", 2012

[18] M. Fornasier and H. Rauhut, "Handbook of mathematical methods in imaging, Compressive sensing", p. 187 - 228, 2011, ISBN 978-0-38792920-0

[19] M. Fornasier and H. Rauhut, "Compressive sensing", 2011, ISBN: 9780387929200

[20] M. Elad, "Sparse and redundant representations: from theory to applications in signal and image processing", 2010, ISBN: 9781441970107

[21] A. C. Fannjiang and T. Strohmer and P. Yan, "Compressed remote sensing of sparse objects",2010

[22] E. J. Candes and M. B. Wakin, "An Introduction to Compressive Sampling" ISSN: 1053-5888

[23] A. Cohen and W. Dahmen and R. DeVore, "Compressed Sensing and Best k-term Approximation", 2009 